\title{
Comunicación interna en los socios de un mercado de la Sierra Central del Perú
}

\author{
Internal communication in the partners of a market in the Central Highlands of Peru
}

\author{
Arón Orlando Cabrera Vega' \\ Pamela Cristina Quijada Farias² \\ Rubén Darío Alania Contreras 3
}

RECIBIDO: 9 DE ABRIL DE 2020

ACEPTADO: 15 DE JUNIO DE 2020

\section{RESUMEN}

La investigación tuvo como objetivo caracterizar la comunicación interna en los socios del Mercado Ramos Toscano de Huancayo - Perú. El estudio fue de enfoque mixto, tipo básico y diseño descriptivo simple, en la etapa cuantitativa se tuvo una muestra de 110 socios del mercado a quienes se administró un cuestionario de

\footnotetext{
1 Egresado de Ciencias y Tecnologías de la Comunicación, Facultad de Humanidades, Universidad Continental, 73005691@continental.edu.pe ORCID. https://orcid.org/0000-0002-0790-6767

Google Scholar: https://scholar.google.com/citations?user=jJl4pbkAAAAJ\&hl=es\&authuser=1 \&oi=ao

2 Egresado de Ciencias y Tecnologías de la Comunicación, Facultad de Humanidades, Universidad Continental, 70051429@continental.edu.pe ORCID. https://orcid.org/0000-0001-8220-8961

Google Scholar: https://scholar.google.com/citations?user=Bv-8W18AAAAJ\&hl=es\&authuser=1

${ }^{3}$ Doctor en Educación, Magister en Docencia en el Nivel Superior, Licenciado en Ciencias de la Comunicación, Psicólogo, Docente, Universidad Continental, Perú; ralania@continental.edu.pe,

Scopus Author ID: 57205187652 ORCID: https://orcid.org/0000-0003-4303-1037,

Google Scholar: https://scholar.google.com/citations? user=4vaclDMAAAAJ\&hl=es\&oi=ao
}

Socialium revista científica de Ciencias Sociales, Vol 4 - No. 2, julio - diciembre 2020, pág. 459-470. 


\section{0}

comunicación interna diseñado para la investigación y en la etapa cualitativa se contó como una muestra de 5 socios del mercado a quienes se realizó entrevistas a profundidad. Como resultado, se halló que el nivel de comunicación interna en el Mercado Ramos Toscano es alto $(66,4 \%)$, predominando canales de comunicación no formales y modos de comunicación espontáneos que posibilitan una la organización eficiente, sobre todo cuando los objetivos son comunes. Se concluyó que la comunicación interna en la unidad de análisis es alta, principalmente debido a la antigüedad de los socios, generando una convivencia que permite una comunicación adecuada, predominantemente no formal, de carácter directo, horizontal, espontánea y potenciada por el respeto a valores del mundo andino como solidaridad y cooperativismo.

Palabras clave: comunicación interna, mercado, socios

\section{ABSTRACT}

The objective of the investigation was to characterize the internal communication in the partners of the Ramos Toscano Market in Huancayo - Peru. The study was of mixed approach, basic type and simple descriptive design, in the quantitative stage a sample of 110 market partners was obtained, to whom an internal communication questionnaire designed for the investigation was administered, and in the qualitative stage counted with a sample of 5 market partners to whom in-depth interviews were conducted. As a result, it was found that the level of internal communication in the Ramos Toscano Market is high (66.4\%), prevailing non-formal communication channels and spontaneous communication modes that enable efficient organization, especially when the objectives are common. It was concluded that internal communication in the analysis unit is high, mainly due to the length of employment of the members, generating a coexistence that allows adequate communication, predominantly non-formal, direct, horizontal, spontaneous and empowered by respect for values of the Andean peoples as solidarity and cooperativism.

Keywords: Internal communication, market, partners. 


\section{Introducción}

Según una macro encuesta online hecha por la revista internacional América Economía y el Centro de Estudios Financieros (CEF) de España (2010), en más de 29 países, se concluyó que la "enfermedad empresarial" que más influye en las empresas es la mala comunicación interna.

En el Perú, las investigaciones sobre comunicación interna en instituciones con estructuras tradicionales son escasas. En la ciudad de Huancayo, se desarrollan muchas instituciones y organizaciones con dinámicas de comunicación que obedecen a hábitos culturales de larga data y donde los enfoques de comunicación organizacional no han intervenido. Al ser una localidad netamente comercial, Huancayo ha tenido un crecimiento exponencial en el último siglo gracias a una actividad comercial basada en las tradicionales ferias, que son el distintivo del origen de la ciudad que funcionan desde épocas previas a la conquista española. En ese periodo de tiempo se han estructurado asociaciones y gremios de comerciantes, que mantienen sus propios modos de comunicación interna.

A pesar de ser un fenómeno con características propias, no se ha encontrado referencias de investigaciones dirigidas a evaluar el aspecto comunicacional de organizaciones tan tradicionales como los mercados en la población de Huancayo, cuyas prácticas reflejan en gran modo formas comunicativas muy difundidas en la propia ciudad. Los mercados de abastos son lugares donde los socios, interactúan a diario con una gran cantidad de clientes, configurando se esa manera su identidad, que es parte primordial de toda organización. Debido a las características culturales de las personas que conformaron la unidad de análisis de la investigación, la comunicación interna del Mercado Ramos Toscano de Huancayo, mantiene una estructura no formal. De acuerdo a lo planteado, objetivo de la investigación fue caracterizar de comunicación interna en los socios del mercado Ramos Toscano de Huancayo.

Los antecedentes, tomados para la investigación, coinciden en definir a la comunicación interna como base fundamental para el desenvolvimiento de una organización, así Laurenti (2017) indica que emplear de manera correcta la comunicación interna otorga muchas ventajas y beneficios ala organización. Al estudiar la gestión de la comunicación interna en la Municipalidad de Ulapes en La 


\section{2}

Rioja - Argentina, identificó la falta de información sobre los beneficios de la comunicación interna y carencia de actualización de los canales comunicativos utilizados. Castro (2012) al investigar la comunicación interna de la Municipalidad Provincial del Santa, Trujillo - Perú, halló que la entidad aún mantiene un estilo de gobierno burocrático con escaso el empoderamiento de los trabajadores y sin una adecuada comunicación interna, la estructura piramidal de la comunicación complicaba la labor de los trabajadores, perjudicando en consecuencia, a los usuarios de los servicios. Villoslada y Calixto (2009) al estudiar las estrategias de comunicación utilizadas por los comités del Vaso de Leche, en el distrito de San Juan de Iscos, Chupaca - Perú, hallaron que se aplicaban estrategias inusuales y a través de medios variados; concluyendo que existía una relación eficaz entre las estrategias de comunicación y la participación ciudadana de los comités de vaso de leche del distrito.

Para entender las características propias de comunicación interna en la unidad de análisis, es necesario partir de los fundamentos de la teoría de los medios a las mediaciones de Martín-Barbero (1987), quien afirma que la cultura es la gran mediadora de todo proceso de producción comunicativa y que el eje de debate de los estudios de la comunicación debe desplazarse de los medios a las mediaciones. El autor define a las mediaciones como "ese lugar donde es posible comprender la interacción entre el espacio de la producción y la recepción" de la comunicación (Martín-Barbero y Muñoz, 1992, p. 20). En ese sentido se entiende la medicación como el proceso de recepción en la comunicación, donde se crean y recrean significados y sentidos desde la interacción de la audiencia con el medio y otras instancias sociales. Para Gonzales (1991) las mediaciones son como especie de ritual publico cultural, donde están presentes todos los actores de la comunidad. Lo que es relevante para un actor es influenciada por las mediaciones sociales, en ese sentido el aspecto importante de la comunicación no es tanto el mensaje sino el proceso de recepción.

Orozco (1997) propuso cuatro grupos de mediaciones: Mediación cultural, que es el proceso cognitivo influenciado por la cultura. Mediación individual que es lo que sucede dentro de la mente del receptor, esta medicación está directamente relacionada con la comunicación intrapersonal. Mediaciones situacionales e 
institucionales, se multiplican de acuerdo a los escenarios en el que se desarrolla, cada escenario incluye posibilidades y limitaciones que no meramente espaciales, esta mediación está relacionada con la comunicación institucional, las mediaciones institucionales actúan como comunidades de interpretación, porque desde ella se interpretan en gran parte de los mensajes percibidos.

Desde el punto de vista corporativo y administrativo el rol de la comunicación interna es destacado por Chiavenato (211) y Tessi (2011), quienes delimitan a la comunicación como una actividad administrativa con dos propósitos fundamentales: infundir las posturas necesarias para generar motivación, participación y satisfacción laboral, y traspasar la información y la aprehensión necesaria para que las personas realicen su trabajo adecuadamente. Según Chiavenato (2007), la comunicación, es uno de los procesos fundamentales del ser humano y la organización social, requiere un código para formular un mensaje y enviarlo en forma de un signo por medio de un canal determinado a un receptor, que lo decodifica y aclara su definición. Sin dejar de lado el manejar la comunicación interna de modo transversal, con estrategias que infieran a los distintos niveles de la organización como emisores de mensajes, y no solo como receptores de información (Tessi, 2011).

Al ser el mercado Ramos Toscano una organización tradicional y cerrada en el acceso a la información, fue complicado en un inicio acceder a la muestra y contar con su colaboración, sin embargo, se persistió, dando a conocer a los socios el consentimiento informado y el anonimato de las encuestas, a lo cual en su mayoría accedieron.

\section{Método}

La investigación fue de enfoque mixto, de tipo básico, diseño no experimental, transaccional, descriptivo simple, pues como refiere Hernández, Fernández y Baptista (2014, p. 155) "Analizar cuál es el nivel o modalidad de una o diversas variables en un momento dado... Su propósito es describir variables y analizar su incidencia e interrelación en un momento dado". 


\section{4}

En la etapa cuantitativa, la población - muestra estuvo constituida por 110 socios que conformaban la organización al momento de realizarse la encuesta.

\section{Tabla 1}

Perfil de los socios $(n=110)$

\begin{tabular}{|c|c|c|}
\hline $\begin{array}{l}\text { Característica y } \\
\text { categorías }\end{array}$ & Socios & $\%$ \\
\hline \multicolumn{3}{|l|}{ Sexo } \\
\hline Femenino & 80 & 72,7 \\
\hline Masculino & 30 & 27,3 \\
\hline \multicolumn{3}{|c|}{ Tiempo de actividad (años) } \\
\hline Menos de 6 & 23 & 20,9 \\
\hline 6 a 10 & 39 & 35,5 \\
\hline Más de 10 & 48 & 43,6 \\
\hline
\end{tabular}

Los socios del mercado Ramos Toscano de la ciudad de Huancayo se caracterizan por ser predominantemente mujeres $(72,7 \%$ ) y el $27,3 \%$ varones. El $43,6 \%$ de los socios se dedican a la actividad comercial en el mercado por más de 10 años, el $35,5 \%$ de 6 a 10 años y el $20,9 \%$ menos de 6 años.

El instrumento de recolección de datos utilizado fue el cuestionario de comunicación interna en los socios de un mercado, diseñado por los autores para la presente investigación, abordando tres dimensiones: comunicación intrapersonal, comunicación interpersonal y comunicación institucional. Se manejó un total de nueve ítems, tres para cada dimensión. Las opciones de respuesta se basaron en la escala de Likert en cinco niveles de respuesta, lo que permitió analizar de manera más precisa los resultados.

Se realizó la validez de contenido, en base al juicio de tres expertos, dos con grado de magíster y uno con grado de doctor especialistas en los campos de comunicación e investigación. Todos los ítems del instrumento de medición presentaron validez buena (61 - $80 \%$ ) o muy buena (81 - 100\%), teniendo como indicadores de evaluación: claridad, objetividad, actualidad, organización, suficiencia, intencionalidad, consistencia, coherencia, metodología, pertinencia. 
En la etapa cualitativa se trabajó con una muestra de 5 socios del mercado Ramos Toscano, tres mujeres y dos varones cuya edad oscilaba entre los 31 a 56 años; el muestreo fue por conveniencia.

A los participantes, se realizó entrevistas a profundidad sobre las siguientes subcategorías: necesidades y modos de comunicación interna, necesidades y modos de comunicación intrapersonal, necesidades y modos de comunicación interpersonal, necesidades y modos de comunicación institucional, valoración de la comunicación interna.

\section{Resultados}

\section{Análisis cuantitativo de la comunicación interna}

\section{Tabla 2}

Nivel de la comunicación interna $(n=110)$

\begin{tabular}{lcc}
\hline Nivel & Socios & $\%$ \\
\hline Bajo & 1 & 0,9 \\
Medio & 36 & 32,7 \\
Alto & 73 & 66,4 \\
\hline
\end{tabular}

La tabla 2 indica que el nivel de comunicación interna de los socios del mercado es alto $(66,4 \%)$, del $32,7 \%$ medio y solo del $0,9 \%$ bajo.

\section{Análisis cuantitativo de las dimensiones de la comunicación interna}

\section{Tabla 3}

Nivel de la comunicación intrapersonal $(n=110)$

\begin{tabular}{lcc}
\hline Nivel & Socios & $\%$ \\
\hline Bajo & 1 & 0,9 \\
Medio & 49 & 44,5 \\
Alto & 60 & 54,5 \\
\hline
\end{tabular}




\section{6}

La tabla 3 muestra que el nivel de comunicación intrapersonal de los socios del mercado es alto $(54,5 \%)$, del $44,5 \%$ medio y solo del $0,9 \%$ bajo.

\section{Tabla 4}

Nivel de la comunicación interpersonal $(n=110)$

\begin{tabular}{lcc}
\hline Nivel & Socios & $\%$ \\
\hline Bajo & 3 & 2,7 \\
Medio & 47 & 42,7 \\
Alto & 60 & 54,5 \\
\hline
\end{tabular}

En la tabla 4 se aprecia que el nivel de comunicación interpersonal de los socios del mercado es alto $(54,5 \%)$, del $42,7 \%$ medio y solo del $2,7 \%$ bajo.

\section{Tabla 5}

Nivel de la comunicación institucional $(n=110)$

\begin{tabular}{lcc}
\hline Nivel & Socios & $\%$ \\
\hline Bajo & 10 & 9,1 \\
Medio & 36 & 32,7 \\
Alto & 64 & 58,2 \\
\hline
\end{tabular}

La tabla 5 muestra que el nivel de comunicación interna de los socios del mercado es alto $(58,2 \%)$, del $32,7 \%$ medio y solo del $9,1 \%$ bajo. 


\section{Análisis cualitativo de la comunicación interna}

\section{Tabla 6}

Entrevista a socios de mercado: Categoría comunicación interna ( $n=5)$

\begin{tabular}{|c|c|}
\hline Subcategoría & Interpretación \\
\hline $\begin{array}{l}\text { Necesidades y } \\
\text { modos de la } \\
\text { comunicación } \\
\text { interna. }\end{array}$ & $\begin{array}{l}\text { Los socios al interactuar a diario con una gran cantidad de clientes y entre ellos, } \\
\text { manifiestan manejo empírico de la comunicación interna debido a la } \\
\text { convivencia rutinaria que manejan los socios por contar de entre } 6 \text { a } 10 \text { años } \\
\text { de antigüedad. Por esta razón los entrevistados indicaron que el trato } \\
\text { comunicacional entre ellos es espontaneo, directo y eficaz en su mayoría. }\end{array}$ \\
\hline $\begin{array}{l}\text { Necesidades y } \\
\text { modos de } \\
\text { comunicación } \\
\text { intrapersonal. }\end{array}$ & $\begin{array}{l}\text { Los manifiestan que tiene interés constante por mantenerse informados } \\
\text { respecto a la organización y a los otros socios, y cuando tiene la necesidad de } \\
\text { trasmitir algo por lo general lo hacen en forma directa y en situaciones que lo } \\
\text { ameritan, nombran lideres informales que transmitieron las posturas de los } \\
\text { colaboradores, manifiestan empoderamiento para mejorar la comunicación } \\
\text { en la institución. }\end{array}$ \\
\hline
\end{tabular}

Necesidades y modos de comunicación interpersonal,

Necesidades y modos de comunicación institucional.

Valoración de la comunicación interna
Generalmente los socios tienen una empatía entre ellos, por la antigüedad de años trabajando en la institución lo que genera una comunicación fluida porque las conversaciones son cotidianas y directas, también esta se refuerza por reuniones semanales entre socios donde se busca una comunicación horizontal, solucionando inconvenientes presentados y guiados de una convivencia no burocrática. La comunicación es fluida.

Los socios del mercado Ramos Toscano buscan mediante votaciones democráticas hacerles frente a los problemas de su centro de trabajo, construyendo una ayuda colectiva para así mantener la solidaridad, uno de valores ya implantados entre ellos, llegando a un consenso para no producir descontentos en sus decisiones. En situaciones de conflicto son los socios de mayor antigüedad quienes intermedian. Cuando se presentas deficiencia comunicacional con del área administrativa lo solucionan a través d ellos líderes informales.

La mayoría de los socios del mercado Ramos Toscano, afirma que existe una iniciativa propia por no tergiversar un mensaje, para así llevar una correcta convivencia en base a cooperación mutua y solidaridad entre sus miembros, la comunicación horizontal facilita a no crear conflictos, siendo esta no formal pero eficaz.

En la tabla 6 se muestra la interpretación cualitativa de las respuestas dadas en la entrevista a profundidad por 5 socios del mercado Ramos Toscano, en torno a la categoría comunicación interna; la interpretación está realizada en base a los conceptos más recurrentes y resonantes de los testimonios. 


\section{8}

\section{Discusión}

El estudio determinó que, según la percepción de los socios, la comunicación interna en el mercado Ramos Toscano es exitosa (66.4\%, nivel alto y $32,7 \%$ nivel medio). En base a los resultados estadísticos se hizo necesario indagar el porqué de la eficacia, siendo una organización donde la comunicación no formal e incluso espontanea es más común que la formal, y los niveles de jerarquía no mantienen la estructura y funcionalidad de otras instituciones.

Tessi (2011), propone que se considere a cualquier organización como un órgano vivo y donde sus integrantes se comunican de una u otra manera, aun así, la organización no intente generar comunicación interna, si lo hace.

Las entrevistas a profundidad realizadas en la etapa cualitativa permitieron dilucidar las interrogantes surgidas (tabla 6), en base a ello es posible afirmar que es el factor cultural de los socios, los años de convivencia y valores muy arraigados, los que facilitan una comunicación fluida y adecuada. La interacción de los socios destaca por su familiaridad, empatía y práctica de valores muy arraigados del mundo andino, como la solidaridad y cooperación aún se mantienen vivos en organización como los tradicionales mercados de abastos, constituyendo un baluarte social y de resistencia ante las nuevas formas de mercadotecnia y comunicación organizacional fomentadas por el etnocentrismo occidental.

En el centro de abastos, los socios con mayor cantidad de años de servicio desarrollan una adecuada comunicación interna ya que su accionar del día a día va de la mano con sus ideas y emociones teniendo claro sus funciones y puesto de trabajo. La receptividad entre los socios es óptima debido a que muestran predisposición y seguridad al hablar con sus compañeros de trabajo. Además, existen espacios que favorecen al encuentro y comunicación con los directivos.

De acuerdo a Martin-Barbero (1987) y Orozco (1997), son las características culturales en sus dinámica individual, grupal e institucional, las que configuran la significancia de los mensajes y por ende la eficacia del proceso comunicacional. En ese sentido el mercado Ramos Toscano se presenta como un microcosmos, con características propias, cuya particular instancia cultural define los significados y sentidos de la comunicación. De acuerdo con Orozco (1997) el mercado Ramos Toscano al estar constituida por un conjunto de sujetos sociales unidos por un ámbito de significación, 
del cual emerge una significación especial para su actuación social, se constituye en una comunidad interpretativa.

La entrevista a profundidad a socios del mercado fue un punto clave para el desarrollo de la investigación porque permitió descubrir aspectos importantes de la comunicación interna, que un estudio solamente cuantitativo podría haber pasado por alto, el incorporar el método cualitativo a la investigación posibilitó profundizar en la característica particular que tiene la dinámica de la comunicación interna del mercado de abastos. En ese sentido se siguiere a los investigadores que abordan la variable de comunicación interna aplicar investigaciones de enfoque misto para profundizar los fenómenos abordados y dar un aporte más trascendente a las ciencias sociales.

Los resultados de la investigación proponen ampliar el panorama en los estudios de la comunicación organizacional y reestructurar aquellos enfoques de influencia sajona, muchas veces alejados de las realidades locales, y volver la mirada a cotidianidad cultural de los pueblos.

Desaprender los verticales esquemas de comunicación que subestiman los métodos de culturas populares, y reaprender a través de la investigación las formas comunicación eficaz, arraigadas en las culturas latinoamericana. Las lecciones aprendidas posibilitaran desarrollar estrategias pertinentes y exitosas, en sintonía con las mediaciones comunicacionales de la población.

\section{Conclusiones}

Se identificó que la comunicación interna en el mercado Ramos Toscano de la ciudad de Huancayo es alta, sobre todo debido a que la mayoría de socios cuenta con más de seis años de servicio en el mercado, generando una convivencia que les permite una comunicación fluida.

La comunicación interna en el mercado Ramos Toscano de Huancayo se caracteriza por ser predominantemente no formal, de carácter directo, horizontal, espontánea y potenciada por el respeto a valores del mundo andino como solidaridad y cooperativismo. 


\section{0}

El éxito de la comunicación interna en el mercado Ramos Toscano se debe a que se practican formas tradicionales de comunicación y convivencia empática.

\section{Referencias}

Castro, G. (2012.). Influencia de la comunicación interna en el mejoramiento de la cultura organizacional de los trabajadores de la Municipalidad Provincial del Santa. Universidad Nacional de Trujillo, Trujillo.

Centro de Estudios Financieros. (2010). CEF. Obtenido de https://www.cef.es/es

Chiavenato, I. (2007). Administracion de Recursos Humanos. Viradouro, Estado de São Paulo, Brasil.

Hernández, R., Fernández, C., yBaptista, P. (2014). Metodología de la investigación. México D.F: McGraw-Hill

Laurenti, V. A. (2017). Gestión de la comunicación interna en la municipalidad de Ulapes la Rioja. Universidad Siglo 21, Córdoba.

Likert, R. (1932). Método de evaluaciones sumarias. Estados Unidos.

Martín-Barbero, J. (1987). De los medios a las mediaciones. Comunicación, cultura y hegemonía. México: Gili.

Martín-Barbero, J. y Muñoz S. (1992). Televisión y melodrama: Géneros y lecturas de la telenovela en Colombia. Bogota: Tercer Mundo.

Orozco, G. (1997). Medios, audiencias y mediaciones. Comunicar. Revista Científica de Comunicación y Educación, 8, 25-30.

Tessi, M. (2011). Comunicación interna en la práctica: : Siete premisas para la comunicación en el trabajo. Granica.

Villoslada Taipe, A., y Calixto Rojas, L. A. (2009). Estrategias de comunicación y participación ciudadana en los comités del vaso de leche del distrito de San Juan de Iscos. Universidad Nacional del Centro del Perú, Huancayo. 